\title{
RELAÇÃO ENTRE DIFERENTES CARACTERES DE PLANTAS JOVENS DE SERINGUEIRA ( $\left.{ }^{1}\right)$
}

\author{
CÉSAR LAVORENTI (2), PAULO DE SOUZA GONÇALVES $(3,6)$, \\ MARIO CARDOSO (4, 6), MARCO MILAN BOAVENTURA (4) \\ E ANTONIO LÚCIOM. MARTINS (5)
}

\begin{abstract}
RESUMO
O presente trabalho foi realizado com o objetivo de determinar a existência e as magnitudes de correlaçōes e regressōes lineares simples em plântulas jovens de seringueira (Hevea spp.), para melhor condução de seleção nos fuluros trabalhos de melhoramento. Foram utilizadas médias de produçäo de borracha seca por plântulas por corte, através do teste Hamaker-Morris-Mann (P); circunferência do caule (CC); espessura de casca (EC); número de anéis (NA); diâmetro dos vasos (DV); densidade dos vasos laticfleros (D) e distáncia média entre anéis de vasos consecutivos (DMEAVC) em um viveiro de cruzamento com três anos e meio de idade. Os resultados mostraram, entre outros fatores, que as correlaçōes lineares simples de $P$ com CC, EC, NA, D, DV e DMEAVC foram, respectivamente, $r$ $=0,61,0,34,0,28,0,29,0,43$ e $-0,13$. As correlaçסes de CC com EC, NA, D, DV e DMEAVC foram: $0,65,0,22,0,37,0,33$ e 0,096 respectivamente. Estudos de regressão linear simples de $P$ com CC, EC, NA, DV, D e DMEAVC sugerem que $C C$ foi o caráter independente mais significativo, contribuindo com $36 \%$ da variação em $P$. Em relação ao vigor, a regressão de $\mathrm{CC}$ com os respectivos caracteres sugere que $E C$ foi 0 único caráter que contribuiu significativamente para a variaçäo de CC com $42 \%$. As altas correlações observadas da produção com circunferência do caule e com espessura de casca evidenciam a possibilidade de obter genótipos jovens de boa capacidade produtiva e grande vigor, através de seleçảo precoce dessas variáveis.
\end{abstract}

Termos de indexaçáo: seringueira, Hevea spp., caracteres anatômicos da casca, correlaçס̄es e regressర̄es lineares.

(1) Recebido para publicaçăo em 26 de outubro de 1989 e aceito em 2 de abril de 1990.

(2) Estagiário do Programa de Seringueira da Divisão de Plantas Industriais (DPI) do Instituto Agronómico de Campinas (IAC), Caixa Postal 28, 13001 Campinas, SP.

(3) EMBRAPA, Programa Integrado de São Paulo. Programa de Seringueira da DPI (IAC).

(4) Programa de Seringueira da DPI (LAC).

(5) Estaçáo Experimental de Pindorama (IAC).

(6) Com bolsa de pesquisa do CNPq. 


\section{ABSTRACT \\ CORRELATIONS AND REGRESSIONS STUDIES AMONG JUVENILE RUBBER TREE CHARACTERS}

This study was undertaken aiming to determine the existence of linear correlations, based on simple regression studies for a better improvement of young rubber tree (Hevea spp.) breeding and selection. The characters studied were: yield of dry rubber per tapping by Hamaker-Morris-Mann test tapping $(P)$, mean gurth $(C C)$, bark thickness (EC), number of latex vessel rings (NA), diameter of latex vesseis (DV), density of latex vesseis per $5 \mathrm{~mm}$ within rings (D) and distance between latex vesseis rings (DMEAVC) in a three and half years old nursery. The results showed that among other characters, the correlation values between $P$ and $C C, E C, N A$, DV, D, DMEAVC were $r=0.61,0.34,0.28,0.29,0.43$ and -0.13 respectively. The correlation coefficients values between $C C$ and EC, NA, DV, D, DMEAVC were: $r=0.65,0.22,0.37,0.33$ and 0.096 respectively. Linear simple regression of $P$ with $C C, E C, N A, D V, D$ and DMEAVC suggested that $\mathrm{CC}$ was the only important and significant character accounting for $36 \%$ of the variation on $\mathrm{P}$. Concerning with vigor, the regression of $\mathrm{CC}$ on the same characters suggest that EC was the only important and significant character accounting for $42 \%$ of the variation on CC. The high correlation of mean girth with yield and bark thickness showed that it is possible to obtain high yielding young ortets through early selection of these characters.

Index terms: rubber tree, Hevea spp, bark anatomic characters, linear correlation and regression.

\section{INTRODUÇĀO}

Em Hevea spp., um dos caracteres mais importantes na seleção de material genético é a produção do látex. A avaliação final de um clone é um processo moroso que exige, normalmente, de dois a três anos, da polinizaçāo à clonagem do material.

Dessa forma, o tempo necessário para produzir e testar novos clones abrange, no mínimo, de 20 a 30 anos, até a recomendação final ao nivel de produtor. Isso tem feito com que melhoristas conduzissem pesquisas para reduzir o ciclo de seleçāo, ou pesquisas envolvendo avaliaçōes de caracteres da planta para estimar-lhes as relaçōes.

O estudo de regressão e correlação entre caracteres fenotípicos é importante, uma vez que, no melhoramento da seringueira, em geral, existe a preocupaçāo de aprimorar o material genético, não para caracteres isolados e, sim, para um conjunto simultâneo de caracteres. Esta seria uma das formas de predizer a produção provável em plantas jovens, reduzindo consideravelmente o tempo necessário para produzir um clone e recomendá-lo para utilização comercial. 
A possibilidade de estabelecer estudos de correlaçōes entre os mais variados caracteres de Hevea spp. têm sido, há algum tempo, objeto de investigação por muitos pesquisadores da seringueira (WHITBY, 1919; BOBILIOFF, 1920; BRYCE \& CAAD, 1924; NARAYANAN et al.,1974). No Brasil, trabaihos de correlações entre a produção e os mais variados caracteres foram desenvolvidos por CALDAS (1977); SIQUEIRA (1978); VALOIS et al. (1978); GONÇALVES et al. (1980a, b); PINHEIRO (1981); PAIVA et al. (1982a, b); RIBEIRO (1983) e, mais recentemente, ALVES (1985).

Esse trabalho foi realizado para determinar a existência e as magnitudes de correlaçōes lineares simples entre caracteres medidos em progênies de famílias de meios-irmãos e irmãos-germanos, em viveiro de cruzamento, objetivando melhor condução nos futuros trabalhos de melhoramento e seleçāo em plântulas de seringueira.

\section{MATERIAL E MÉTODOS}

O trabalho foi desenvolvido a partir de dados de produção de borracha seca, obtidos através do teste Hamaker-Morris-Mann (HMM) modificado (TAN \& SUBRAMANIAN, 1976) e caracteres anatômicos da casca e circunferência do caule em plântulas de viveiro de cruzamento estabelecido em 1985 na Estação Experimental do Instituto Agronômico em Pindorama, com altitude de $546 \mathrm{~m}$, temperatura média anual de $22^{\circ} \mathrm{C}$ e precipitação pluvial média de $1.328 \mathrm{~mm}$. O solo predominante é o podzólico vermelho-amarelo TB, eutrófico, textura arenosa, abrupto, fase relevo ondulado.

$O$ viveiro de cruzamento foi estabelecido no espaçamento de $1,5 \times 1,5 \mathrm{~m}$ e as plântulas, agrupadas em famílias, originaram-se de sementes obtidas através de polinizaçōes controladas (famílias de irmãos-germanos) e de polinizações abertas (famílias de meios-irmãos), na coleçāo de clones de seringueiras do Instituto Agronômico, localizada no Centro Experimental de Campinas (CEC).

Para efeito de seleção, no terceiro ano de desenvolvimento vegetativo das plântulas, efetuaram-se mensuraçōes de circunferência do caule a 0,5m de altura do solo. No mesmo periodo, as plântulas foram submetidas ao teste HMM, mediante uma faca do tipo "jebong", empregada na sangria normal; as árvores foram sangradas a $0,20 \mathrm{~m}$ de altura do solo no sistema $\mathrm{s} / 2 \mathrm{~d} / 2$ (meia espiral em dias alternados). Efetuaram-se 30 sangrias para que a produção fosse tomada cumulativamente plântula por plântula, através da coagulação natural do látex nos recipientes de coleta. Para quantificação da borracha seca, adotou-se o método rápido de determinação dos sólidos totais, pelo qual os "biscoitos" correspondentes a cada plântula foram secos à sombra, em ambiente ventilado e por 2-3 meses até alcançar peso constante. Da média dos 30 cortes, foram obtidos dados de produção de sangria por plântula, expressos em gramas de borracha seca.

Os caracteres anatômicos foram determinados nas amostras da seguinte forma: 
1. Espessura de casca virgem, tomada a $0,50 \mathrm{~m}$ de altura do caule de cada plântula, com o auxílio de um "vasador", e medida com um paquímetro em milímetros.

2. Número total de anéis de vasos laticiferos, determinado pelo exame das seçóes radiais longitudinais.

3. Diâmetro dos vasos laticíferos, observado através da seção transversal.

4. Densidade dos vasos laticiferos em $5 \mathrm{~mm}$ do anel, estimada pela densidade da média de todos os anéis.

5. Distância média entre anéis de vasos consecutivos, estimada com base em todos os anéis.

Os caracteres anatômicos referentes à casca da seringueira foram analisados e estimados em laboratório do Centro de Pesquisa Agroflorestal da Amazônia (CPAA), da EMBRAPA, situado em Manaus, AM.

Os dados referentes ao número de anéis foram transformados pela expressão $\sqrt{x i+0,5}$, de acordo com STEEL \& TORRIE (1960).

Determinararn-se os coeficientes de correlação de suas significâncias verificadas pelo teste " $\mathrm{t}$ " ao nivel de $5 \%$ e estimaram-se os coeficientes de regressão linear simples, utilizando-se as médias dos caracteres de cada indivíduo da população de plântulas.

\section{RESULTADOS E DISCUSSÃO}

No quadro 1, encontram-se médias, intervalo de variação, desvio padrăo, coeficiente de variação e número de plântulas referentes aos sete caracteres em estudo.

QUADRO 1. Estimativa da média (X), intervalo de variação (IV), desvio padrão (s) e coeficiente de variação $(\mathrm{CV} \%)$ referentes a sete caracteres estudados em uma populaçāo de plântulas de seringueira de quatro anos de idade, cultivadas em viveiro de cruzamento em Pindorama, SP

\begin{tabular}{|c|c|c|c|c|c|c|}
\hline Caracteres & Unidade & $\bar{x}$ & IV & $\mathbf{s}$ & CV\% & $\begin{array}{l}\text { Número de } \\
\text { plântulas }\end{array}$ \\
\hline Produçăo (g/p/corte) & $g$ & 0,57 & $0,03-3,31$ & 0,68 & 119,30 & 56 \\
\hline Circunferência do caule & $\mathrm{cm}$ & 24,70 & $12,00-45,00$ & 5,97 & 24,17 & 58 \\
\hline Espessura da casca & $\mathrm{mm}$ & 3,10 & $1,50-5,20$ & 0,62 & 20,00 & 54 \\
\hline Nümero de anéis & un. & 2,42 & $1,87-2,91$ & 0,36 & 11,15 & 54 \\
\hline Diâmetro dos vasos & $\mu$ & 12,16 & $10,26-14,82$ & 0,96 & 7,90 & 54 \\
\hline $\begin{array}{l}\text { Densidade } \\
\text { Distáncia média entre }\end{array}$ & - & 61,24 & $49,12-78,94$ & 7,16 & 11,69 & 54 \\
\hline Anéis de vasos consecutivos & $\mu$ & 297,96 & $166,44-456,00$ & 78,51 & 26,34 & 54 \\
\hline
\end{tabular}

(") g/p/corte = gramas por plántula por corte de borracha seca; un. = unidade. 
O quadro 2 apresenta os coeficientes de correlação (i) entre os diversos caracteres estudados e respectivas significâncias estatisticas obtidas de acordo com CAMPOS (1984).

Os resultados das análises de regressão linear simples entre a produção e os caracteres anatómicos, entre o vigor, expresso pela circunferência do caule, e esses mesmos caracteres, acham-se no quadro 3, bem como os valores dos coeficientes de determinação $\left(R^{2}\right)$ obtidos nessas análises.

\subsection{Correlaçăo linear simples}

\subsubsection{Produção}

Entre os caracteres estudados, a produção foi o mais variável, com amplitude de variação de 0,03 a 3,31g de borracha seca, e coeficiente de variação de $119,30 \%$ de magnitude, indicando alta variabilidade, importante para a seleção em um programa de melhoramento genético. Esses resultados se assemelham aos obtidos por TAN et al. (1975) e GONÇALVES (1982) em progênies de cruzamento intra- $\mathrm{e}$ interespecíficos respectivamente.

Correlação entre produção e circunferência do caule $\left(r=0,60519^{*}\right)$ toi alta e significativa, confirmando resultados encontrados por NARAYANAN et al. (1974); TAN et al. (1976); RIBEIRO (1983) e, mais recentemente, por ALVES (1985). Produção também foi correlacionada com número de anéis de vasos laticíferos $\left(r=0,28357^{*}\right)$. Tém sido obsenvados coeficientes altos, superiores a $r=0,80 \mathrm{em}$ árvores dentro de um genótipo (clone), e, entre genótipos, magnitudes de coeficientes entre 0,35 e 0,57 (NARAYANAN et al., 1974; TAN et al., 1976; PAIVA, 1980, e GOMES, 1982), concordantes com o resultado do presente trabalho.

Densidade dos vasos laticiferos $\left(r=0,43160^{*}\right)$ foi significativamente correlacionada com produção. Pelo grau de significância, tal resultado sugere que a seleção para este caráter poderá contribuir para o programa de melhoramento genético da seringueira, visando incrementar a produçăo.

\subsubsection{Circunferência do caule}

Em seringueiras, produção e vigor estāo inter-relacionados e, segundo Paardekooper, citado por $\mathrm{HO}$ et al. (1943), mais da metade da variação na produção dos clones é devida às diferenças de circunferência do caule. Entretanto, a precocidade de produção e de vigor somente é possível em uma árvore que se desenvolve vigorosamente na fase juvenil (SIMMONDS, 1989). Há evidência experimental de que o vigor esteja positivamente correlacionado com o desenvolvimento da circunferência do caule (EVERS et al.,1960). 


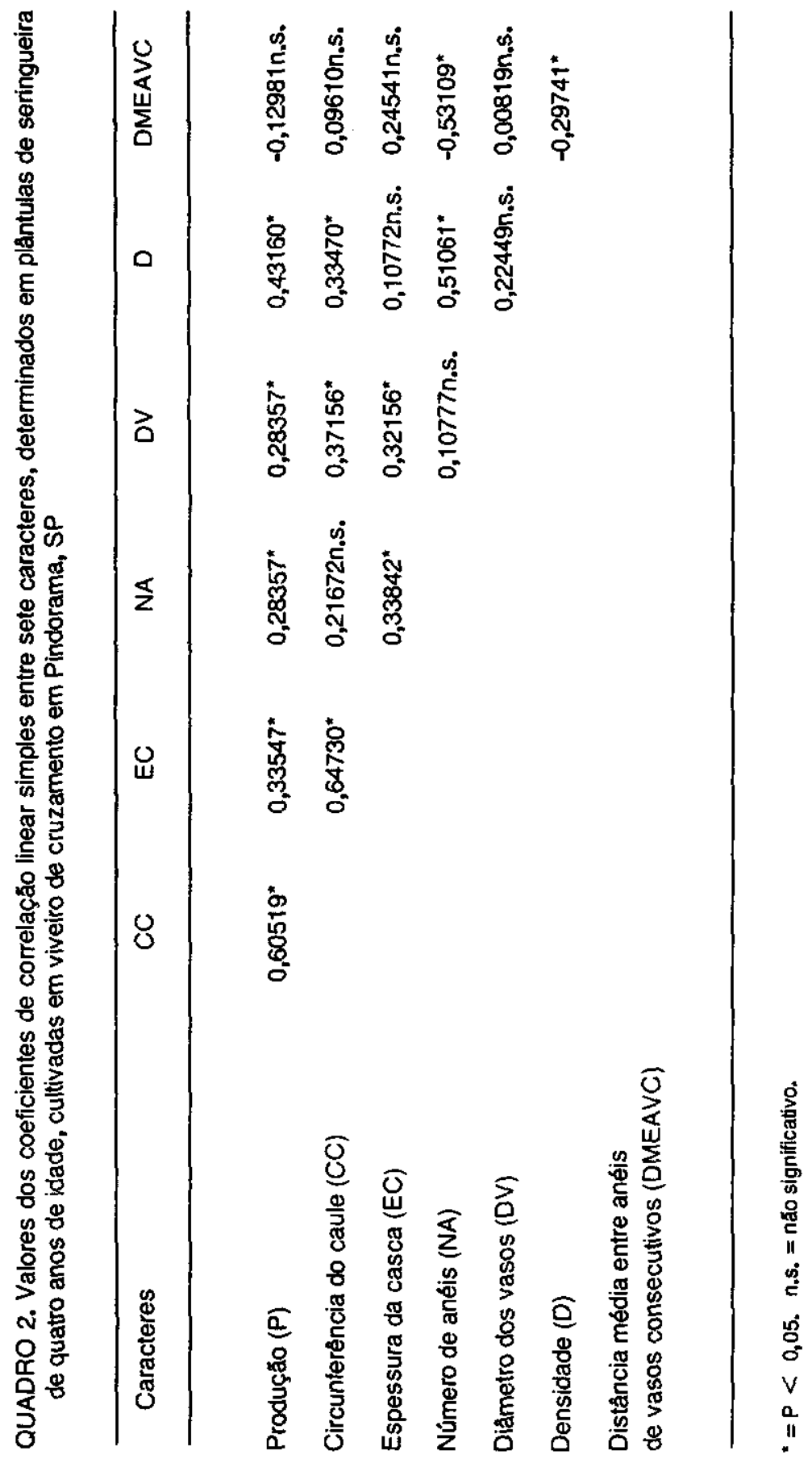


QUADRO 3. Parâmetro das equaçðes de regressáo linear $Y=a+b x$ e coeficientes de determinaçăo $\left(R^{2}\right)$, referentes a plântulas de seringueira cultivadas em viveiro de cruzamento estabelecido em Pindorama, SP

\begin{tabular}{|c|c|c|c|c|c|}
\hline Equação & $\begin{array}{c}\text { Variável } \\
\text { dependente }\end{array}$ & $\begin{array}{c}\text { Variável } \\
\text { independente }\end{array}$ & b & a & $\mathrm{R}^{2}$ \\
\hline 01 & $P$ & $\mathrm{CC}$ & $\begin{array}{r}0,16669^{*} \\
(0,60989)\end{array}$ & 22,27804 & 0,36505 \\
\hline 02 & $\mathrm{P}$ & EC & $\begin{array}{c}0,00925^{*} \\
(0,07654)\end{array}$ & 2,99272 & 0,11254 \\
\hline 03 & $P$ & NA & $\begin{array}{r}0,00443^{*} \\
(0,03161)\end{array}$ & 2,33691 & 0,14711 \\
\hline 04 & $P$ & DV & $\begin{array}{r}0,01309^{*} \\
(0,13077)\end{array}$ & 11,95312 & 0,08041 \\
\hline 05 & $P$ & $D$ & $\begin{array}{c}0,14578^{*} \\
(0,90000)\end{array}$ & 58,95130 & 0,18627 \\
\hline 06 & $P$ & DMEAVC & $\begin{array}{c}-0,49477 n s \\
(11,32567)\end{array}$ & 308,03672 & 0,01685 \\
\hline 07 & $\mathrm{CC}$ & EC & $\begin{array}{r}0,06968^{*} \\
(0,07680)\end{array}$ & 1,35053 & 0,41900 \\
\hline 08 & $\mathrm{CC}$ & NA & $\begin{array}{l}0,00932 \mathrm{~ns} \\
(0,03380)\end{array}$ & 2,16521 & 0,04696 \\
\hline 09 & $\mathrm{CC}$ & DV & $\begin{array}{c}0,06144^{*} \\
(0,12312)\end{array}$ & 10,60911 & 0,13806 \\
\hline 10 & $\mathrm{CC}$ & $D$ & $\begin{array}{c}0,41440^{*} \\
(0,93562)\end{array}$ & 50,77424 & 0,11202 \\
\hline 11 & $\mathrm{CC}$ & DMEAVC & $\begin{array}{c}1,31686 \mathrm{~ns} \\
(10,83627)\end{array}$ & 264,72469 & 0,00924 \\
\hline
\end{tabular}

$P=$ produçáo em gramas de borracha seca por conte obtida atavés do teste $\mathrm{HMM}$ modificado; $\mathrm{CC}=$ circunferéncia do caule; EC = espessura da casca; NA = nưmero de anéis de vasos laticfieros; DV = diâmetro dos vasos laticfieros; $D=$ densidade dos vasos laticfieros; DMEAVC = distáncia média entre anéis de vasos consecutivos. " $=P<0,05$. ns $=$ nấo significativo. Valores dentro dos parénteses correspondem ao erro padráo dos respectivos coeficientes de correlaçăo. 
No presente estudo (Quadro 1) foi observada grande variaçăo expressa através do coeficiente de variação ( $C V=24,17 \%$ ) e do intenalo de variação ( $\mathrm{N}=$ $12,00-45,00)$ em face das influências ambientais e das diferenças genéticas entre as plântulas. No quadro 2 - correlaçōes lineares entre circunferência do caule e vários caracteres morfológicos - encontra-se alto coeficiente de correlaçào entre circunferência do caule,e espessura da casca $\left(r=0,64730^{*}\right)$, indicando que, quanto maior a circunferência do caule, maior a espessura da casca. Resultados semelhantes foram obtidos por NARAYANAN et al. (1973) e GONÇALVES et al. $(1980 a, b)$. Encontraram-se também correlaçōes entre circunferência e diâmẹtro dos vasos $\left(r=0,37156^{*}\right)$ e entre circunferência e densidade $\left(r=0,33470^{\star}\right)$.

\subsubsection{Espessura da casca}

Segundo SIMMONDS (1989), uma árvore de casca espessa torna-se mais fácil de ser sangrada e tem maior número de vasos laticíferos do que uma de casca fina. Partindo dessa hipótese, NARAYANAN et al. (1974) observaram que espessura de casca, diâmetro dos vasos e número de anéis de vasos laticiferos estão inter-relacionados em seringueiras adultas e correlacionados com produção. No material em estudo, aos quatro anos de idade, os valores extremos das plantas variaram de 1,50 a $5,20 \mathrm{~mm}$ para o caráter espessura da casca, apresentando para todas as plântulas uma média de 3,1 mm (Quadro 1).

O resultado da correlaçäo entre espessura da casca e diâmetro dos vasos $\left(r=0,32156^{*}\right)$ confirma os resultados de NARAYANAN et al. (1974) para esse caráter em clones adultos de seringueira.

A correlação entre espessura da casca e número de anéis de vasos laticiferos apresentou valor baixo $\left(r=0,33842^{*}\right)$, concondando com os resultados obtidos por GONÇALVES et al. (1984). De acordo com WEBSTER \& PAARDEKOOPER (1989), a correlação entre esses dois caracteres é muito maior dentro de um genótipo (clone) do que entre diferentes genótipos.

\subsubsection{Número de anéis de vasos laticiferos}

Segundo WEBSTER \& PAARDEKOOPER (1989), tanto a espessura da casca virgem como o número de anéis de vasos laticiferos aumentam com a idade da árvore. $O$ número de anéis é um caráter clonal, mas a freqüência com que eles se originam através do câmbio da planta também depende da taxa de crescimento da árvore, a qual é influenciada por fatores como densidade de plantio e estado nutricional da planta, assim como do próprio clone.

Em seringueiras adultas, o número de vasos laticiferos é um caráter determinante de produção (HO et al., 1973). Segundo o ANNUAL REPORT 1966 
(1967), a maior parte da variação de produçāo dos clones é devida ao número de anéis de vasos laticíferos e circunferência do caule. Isso foi confirmado no presente trabalho, quadro 2, tendo em vista a correlação entre número de anéis e produção $\left(r=0,28357^{*}\right)$. Verificou-se correlação positiva $\left(r=0,51061^{*}\right)$ entre número de anéis e densidade dos vasos, correlação significativa e negativa entre número de anéis e distância média entre anéis de vasos consecutivos $\left(r=-0,53109^{*}\right)$.

\subsubsection{Densidade dos vasos laticíferos}

A densidade de vasos dentro dos anéis, ou seja, o número de vasos por milímetro de anel, varia entre genótipos, porém é sempre maior nos anéis próximos ao câmbio.

A densidade média para todas as plântulas foi de 61,24 , levando-se em consideração o anel mais próximo ao câmbio, obtendo-se valores extremos de 49,12 a 78,94 (Quadro 1). Segundo HO et al. (1973), a densidade dos vasos laticíferos dentro dos anéis é um caráter importante do sistema laticífero.

Correlação significativa e negativa foi obtida entre densidade e distância média entre anéis de vasos consecutivos $\left(r=-0,29471^{\star}\right)$.

\subsection{Regressāo linear simples}

No quadro 3, são mostrados parâmetros das equaçōes de regressāo linear simples com os respectivos coeficientes de determinação $\left(R^{2}\right)$.

Dois grupos de equações de regressão linear simples foram obtidos, conforme se considerou a produçăo $(P)$ ou a circunferência do caule $(\mathrm{CC})$, como variável dependente.

Para a variável dependente $P$ (Quadro 3 ), os valores de b positivos e significativos mostraram que circunferência do caule, densidade e número de anéis de vasos laticiferos contribuiram significativamente com 36,5, 18,6 e $14,7 \%$ respectivamente, na variação de $P$, enquanto as variáveis espessura de casca e diâmetro dos vasos laticíferos, embora significativas, pouco contribuíram.

As equaçōes 07 a 11, referentes ao vigor, representado pela variável dependente $\mathrm{CC}$, revelaram que espessura da casca, diâmetro e densidade dos vasos laticiferos contribuiram com 42,14 e $11 \%$, respectivamente, para sua variação.

\section{CONCLUSÕES}

1. Correlações simples entre produção e circunferência do caule, espessura da casca, número de anéis, diâmetro e densidade dos vasos laticíferos, 
foram positivas e significativas, sugerindo que a seleção para cada um desses caracteres poderá contribuir para um programa de melhoramento genético, visando incrementar a produção.

2. Circunferência do caule e espessura da casca apresentaram correlação positiva e altamente significativa, indicando que a seleção para vigor pode basear-se na espessura da casca.

3. A circunferência do caule contribuiu com $36 \%$ da variaçāo da produção e a espessura da casca com $41 \%$ da variação da circunferência do caule, constituindo, assim, dois caracteres úteis para orientar a seleção para produçāo e vigor.

\section{REFERÉNCIAS BIBLIOGRÁFICAS}

ALVES, R.M. Avaliação precoce de clones primários de seringueira (Hevea spp.) em Belém, PA. Piracicaba, ESALQ, 1985. 156p. Tese (Mestrado)

ANNUAL REPORT 1966. Kuala Lumpur, Malaya, Rubber Research Institute of Malaya, 1967. P.25.

BOBILIOFF, W. Correlation between yield and number of latex vessel rows of Hevea brasiliensis. Archief Rubbercultur Nederlandsch Indie, Batavia, 4:383-391, 1920.

BRYCE, C. \& CAAD, C.H. Yield and growth in Hevea brasiliensis. Ceylon, Department of Agriculture, 1924. (Bulletin, 68)

CALDAS, R.C. Comportamento de clones de seringueira (Hevea spp.) no Estado da Bahia. Piracicaba, ESALQ, 1977. 66p. Tese (Mestrado)

CAMPOS, H. Estatística aplicada à experimentação com cana-de-açúcar. Piracicaba, Fundação de Estudos Agrários Luiz de Queiroz, 1984. 292p.

EVERS, E.; VERBEKE, R. \& MAERTENS, C. Relations entre climat, la phénologie e la production de l'Hevea. Bruxelles, INEAC, 1960. 71p. (INEAC. Série Scientifique, 84)

GOMES, I.B. Anatomy of Hevea and its influence on latex production. Kuala Lumpur, Malaysian Rubber Research and Development Board, 1982. 50p. (Monography, 7)

GONÇALVES, P.S. Collection of Hevea materials from Rondonia Territory in Brazil: a preliminary study. Pesquisa Agropecuária Brasileira, Brasflia, 17(4):575-582,1982.

; ROSSETTI, A.G.; VALOIS, A.C.C.; \& VIEGAS, I.J. Comportamento, estudo de correlaçōes e herdabilidade de alguns caracteres quantitativos em clones jovens de seringueira (Hevea spp.). In: SEMINÁRIO NACIONAL DE SERINGUEIRA, 3., Manaus, 1980. Anais. Brasnia, SUDHEVEA, 1980a. v.1, p.386-410. sileira de Genética, Ribeirão Preto, 7(1):95-107, 1984.

; VASCONCELOS, M.E.C.; VALOIS, A.C.C. \& SILVA, E.C. Herdabilidade, correlaçరes genéticas e fenottpicas de algumas caracter(sticas de clones jovens de seringueira. Pesquisa Agropecuária Brasileira, Brasllia, 15(2):129-136, $1980 \mathrm{~b}$. 
HO, C.Y.; NARAYANAN, R. \& CHEN, K.T. Clonal nursery studies in Hevea; I. Nursery yields and associated structural characteristic and their variations. Journal of the Rubber Research Institute of Malaya, Kuala Lumpur, 23(4):305-316, 1973.

NARAYANAN, R.; GOMEZ, J.B. \& CHEN, K.T. Some structural factors affecting the productivity of Hevea brasiliensis: II. Correlation studies between structural factors and yield. Journal of the Rubber Research Institute of Malaya, Kuala Lumpur, 23:285-297, 1973.

; HO, C.Y. \& CHEN, K.T. Clonal nursery studies in Hevea: III. Correlations between yield, structural characteres latex constituints and plugging index. Journal of the Rubber Research Institute of Malaya, Kuala Lumpur, 24:1-14, 1974.

PAARDEKOOPER, E.C. \& SAMOSORN, S. Clonal variation in latex flow pattern. Journal of the Rubber Research Institute of Malaya, Kuala Lumpur, 23(3):264-272, 1969.

PAIVA, J.R. Estimativas de paråmetros genéticos em seringueira (Hevea spp.) e perspectiva de melhoramento. Piracicaba, ESALQ, 1980. 92p. Tese (Mestrado)

; MIRANDA FILHO, J.B.; SIQUEIRA, E.R. \& VALOIS, A.C.C. Predição do ganho genético de alguns caracteres em seringueira em três esquemas de seleção. Pesquisa Agropecuánia Brasileira, Brasnia, 17(11):1647-1653, 1982a.

; ROSSETTI, A.C. \& GONÇALVES, P.S. Uso do coeficiente de caminhamento no melhoramento da seringueira. Pesquisa Agropecuária Brasileira, Brasnia, 17(3):433-440, $1982 \mathrm{~b}$.

PINHEIRO, F.S.V. Comportamento de alguns clones amazónicos de seringueira (Hevea spp.) nas condiçóes ecológicas de Açailandia: resultados preliminares. Viçosa, UFV, 1981. 83p. Tese (Mestrado)

RIBEIRO, S.I. Comportamento de clones de seringueira (Hevea spp.) em Porto Velho. Lavras, ESAL, 1983. 59p. Tese (Mestrado)

SIMMONDS, N.W. Rubber breeding. In: WEBSTER, C.C. \& BAULKWILL, W.J., eds. Rubber. London, Longman, 1989. cap.3, p.85-124.

SIQUEIRA, E.T. Estimativas de parámetros genéticos de seringueira (Hevea spp.) em condiçóes de viveiro. Viçosa, UFV, 1978. 34p. Tese (Mestrado)

STEEL, R.G.D. \& TORRIE, J.H. Principles and procedures of statistics. New York, MacGraw Hill, 1960. 461p.

TAN, H.; MUKHERJEE, T.K. \& SUBRAMANIAN, S. Estimates of genetic parameters of certain characters in Hevea brasiliensis. Theoretical and Applied Genetics, Berlin, 46:181-190, 1975.

\& SUBRAMANIAN, S. A five-parent diallei cross analyses for certain characters of young Hevea seedlings. In: INTERNATIONAL RUBBER CONFERENCE, Kuala Lumpur, 1975. Proceedings. Kuala Lumpur, 1976. v.2, p.13-16.

VALOIS, A.C.C.; PINHEIRO, E.; CONCEIÇĀO, H.E.O. \& SILVA, M.N.C. Competição de porta-enxertos de seringueira (Hevea spp.) e estimativas de parâmetros genéticos. Pesquisa Agropecuária Brasileira, Brasnia, 13(2):49-54, 1978.

WEBSTER, C.C. \& PAARDEKOOPER, E.C. The botany of the rubber tree. In: WEBSTER, C.C. \& BAULKWILL, W.J., eds. Rubber. London, Longman, 1989. cap.2, p.57-84.

WHITBY, S. Variation in Hevea brasiliensis. Annais of Botany, London, 33:313-321, 1919. 